\title{
Jalons pour une philologie du discours
}

\author{
Jérôme Lecompte ${ }^{1, *}$ \\ ${ }^{1}$ IRET, Université Sorbonne Nouvelle, Maison de la recherche, 4, rue des Irlandais, 75005 Paris
}

\begin{abstract}
Résumé. À partir du constat de Rastier et Adam d'un «déficit philologique » en analyse du discours $(2001 ; 2015)$, nous proposons de coordonner la philologie, la rhétorique et l'analyse du discours dans une méthode conçue pour renouveler l'approche des documents et textes anciens : restituer la configuration discursive à l'intérieur de laquelle le discours a été produit ou re-produit, identifier les informations contextuelles pertinentes lors de la réception doivent permettre de recontextualiser le discours, et de mieux en saisir les enjeux. Trois cas ont été retenus pour l'intérêt et la complexité de leur énonciation, à différents niveaux : empruntés à La Mesnardière, Racine, La Bruyère, ils montreront que cette méthode interdisciplinaire peut appréhender le fonctionnement discursif des rhétoriques de l'âge classique, et ainsi leur reconfiguration à des fins nouvelles (esthétiques, politiques, morales, religieuses).
\end{abstract}

\begin{abstract}
Milestones for a discourse philology. Based on the observation of a "philological deficit" in discourse analysis (Rastier 2001; Adam 2015), we propose to coordinate philology, rhetoric and discourse analysis in a method designed to renew the approach of old documents and texts: restoring the discursive configuration within which the speech was produced or re-produced, identifying the relevant contextual information during the reception should make it possible to recontextualize the speech, and better understand the issues. Three cases were selected for the interest and complexity of their enunciation: borrowed from La Mesnardière, Racine and $\mathrm{La}$ Bruyère, they will show that an interdisciplinary method can apprehend the discursive functioning of rhetorics in the classical age, and their reconfiguration for new purposes (aesthetic, political, moral, religious).
\end{abstract}

Avec la sacralisation du texte, la littérature a fini par rejeter dans l'oubli cette oralité primordiale que les productions de l'âge classique devaient à la prépondérance des modèles de l'éloquence et de la conversation. Ces œuvres la rappelaient pourtant par la vigueur de leur rhétorique, inséparable d'une discursivité forte qui semble fonctionner encore à demi quand on les entend prononcer ou déclamer. Mais redire n'est jamais plus dire à l'identique. Les éléments contextuels qui sous-tendent le discours se dégradent et se perdent, même si leurs traces subsistent parfois, discrètes ou imperceptibles, dans le texte. Non pas que la valeur du discours originel se perde toujours : mais il y a fort à parier que, si elle s'est maintenue, elle a changé. L'éloignement temporel n'a d'ailleurs pas besoin d'être bien grand pour que le contexte fausse plus ou moins compagnie au discours; l'ironie ou

\footnotetext{
*1ecomptejerome@gmail.com
} 
l'ambivalence d'un énoncé reposent déjà sur la possibilité de ces lacunes ; à peine transcrit, le texte d'un discours confronte son lecteur à l'affaiblissement des voix (KerbratOrecchioni, 2015 : 29). Ce sont les dynamiques du discours qui s'éteignent.

Pour empêcher ce refroidissement, la rhétorique animait le discours par des tours de langage énergiques. Dans le Traité sur le sublime traduit par Boileau, le pseudo-Longin estime que, par l'interrogation, « l'auditeur est adroitement trompé, et prend les discours les plus médités pour des choses dites sur l'heure et dans la chaleur $»{ }^{1}$. Figure du discours par excellence, l'interrogation suivie de sa réponse a été préparée par l'orateur: sa prononciation n'est qu'un simulacre d'énonciation faisant illusion sur le public, et l'on est aujourd'hui moins enclin à sentir ce style sublime que les artifices rhétoriques sur lesquels il repose, tandis que Boileau peut encore dire d'Homère qu'une « heureuse chaleur anime ses discours $»^{2}$. Cela ne signifie pas que le contexte soit alors pleinement ressaisi, mais que l'environnement socio-discursif en favorise une réactualisation. Pour mieux entendre la rhétorique, ne faudrait-il pas tenter de retrouver cette « chaleur » perdue ? Mais atteindre le discours dans les textes classiques rend nécessaire un double effort de contextualisation : il faut restituer d'une part la configuration discursive à l'intérieur de laquelle le discours a été produit ou re-produit, et identifier d'autre part les informations contextuelles pertinentes à première réception. Ces deux opérations indispensables à l'analyse du texte comme discours demandent une approche interdisciplinaire, qui permette de coordonner les apports de la philologie, de la rhétorique et de l'analyse de discours.

Quoique l'idée d'une méthode fondée sur la coopération de disciplines aux antagonismes séculaires soulève inévitablement des problèmes épistémologiques, elle fait aussitôt entrevoir un bénéfice réel pour l'analyse des textes et documents anciens, sans condition de littérarité. Avec Aristote, Cicéron, Quintilien, ou Longin, on peut se munir des meilleurs viatiques pour aborder le $\mathrm{XVII}^{\mathrm{e}}$ siècle, puisque leurs rhétoriques n'ont pas cessé alors d'être méditées; mais tout vade-mecum réduit à des nomenclatures fait courir le risque de textualiser la rhétorique, c'est-à-dire de refroidir le discours et d'en fragiliser le souffle. En outre, la reconstitution d'une formation discursive n'empêchera pas que l'acuité de notre regard ne soit diminuée par des manques, déjouée par des masques, et cependant accrue par notre position en surplomb. La rhétorique n'a donc pas intérêt à être refermée sur ellemême, et l'on peut penser qu'elle gagne à s'ouvrir aux apports des sciences du discours (Amossy 2015 : 57). Il semble toutefois difficile d'appréhender le fonctionnement discursif des rhétoriques de l'âge classique et leurs reconfigurations à des fins nouvelles (esthétiques, épistémiques, morales, politiques...) sans un examen philologique préalable. Si l'analyse $\mathrm{du}$ discours sait identifier les savoirs pertinents quand les interactions relèvent d'unités encore accessibles de plain-pied à l'analyste, en revanche elle ne peut atteindre un discours qui serait le produit d'une configuration discursive ancienne et de paramètres énonciatifs perdus pour l'histoire : il faut chercher, par la philologie, à réhabiliter de tels contextes.

Transdisciplinaire par nature, la philologie se prête de mauvais gré à toute tentative de définition : c'est un lieu commun d'avouer d'abord qu'elle y échappe, quand elle ne nous laisse pas en fin de compte le soupçon de l'avoir mal cernée. Cette science de l'établissement et de l'étude des textes a longtemps opposé son objet au discours. Or un texte n'est ni stable, ni autonome ; il matérialise imparfaitement un discours qui, selon les types et les genres, se sera plus ou moins pris dans ses fibres. Il semble donc que la philologie, jadis « intuitivement comprise comme entreprise de contextualisation des énoncés » (Maingueneau : 2005), soit en mesure de contribuer à l'analyse du discours. On répondrait ainsi au constat du « déficit philologique » des sciences du discours dressé par François Rastier (2001: 73) et JeanMichel Adam (2015: 25).

Si l'objet de l'analyse du discours n'est « ni l'organisation textuelle ni la situation de communication, mais ce qui les noue à travers un certain dispositif d'énonciation » (Maingueneau 2012: §17), celui d'une philologie du discours consistera précisément à réparer ces liens rompus par la distance temporelle ou l'affaiblissement des voix. Mais 
comment élucider les contextes et ranimer ainsi le discours en dépôt dans le texte? Il faut conjointement recourir aux moyens propres de la philologie (paléographie, codicologie, bibliologie), de la linguistique textuelle et de l'analyse du discours. D'une discipline à l'autre, on fera saillir la présence de la rhétorique, ce que sa diversité même autorise : elle sera tantôt envisagée comme révélatrice d'une configuration discursive (perspective historique), tantôt employée comme outil de l'analyse du fonctionnement discursif (perspective argumentative). À l'opposé d'une conception fermée du texte induite par une rhétorique structurale, il s'agit de révéler un texte traversé par le discours, en identifiant ce que le contexte dépose en lui, et qui n'est plus forcément perçu.

Trois cas empruntés au XVII ${ }^{\mathrm{e}}$ siècle serviront de premiers jalons à cette méthode. La Mesnardière dans sa traduction du Panégyrique de Trajan de Pline, Racine dans Athalie, et La Bruyère dans un caractère, ont en commun de mettre en œuvre le style élevé, sinon de chercher le sublime, ce qui signifie qu'ils supposent une chaleur qui, pour nous, n'est sans doute plus sensible dans le texte parce qu'elle tient davantage au discours. Traduire, citer, imiter : chaque auteur illustrera une opération qui relie son œuvre à un autre discours, motivant ainsi une analyse philologique et rhétorique attentive à ces relations ${ }^{3}$.

\section{Traduire : une paraphrase « enthousiaste » du Panégyrique de Trajan de Pline le jeune (1638)}

L'orateur doit $[\ldots]$ s'élever, s'exalter, parfois même s'échauffer, s'emporter et souvent frôler l'abîme, car hauteurs et sommets bordent généralement les précipices. Plus sûr est le chemin de la plaine, mais il est plus plat et plus bas [...].

Pline, Lettres, IX, $2^{4}$

De cette chaleur nécessaire à l'éloquence, que subsiste-t-il dans le témoin ? Ainsi du Panégyrique de Trajan d'abord prononcé par Pline le jeune devant le Sénat le $1^{\mathrm{e}}$ septembre 100 , à sa prise de fonction comme consul, puis remanié pour être publié : le discours a été fondu dans un texte dont la pompe impériale fait sentir la froideur et la dureté d'un style d'apparat. Comment rendre dans une autre langue cette chaleur doublement perdue ? Parmi les quatre traductions tentées au XVII ${ }^{\mathrm{e}}$ siècle, l'une retient l'attention parce qu'elle relève ce défi : protégé de Richelieu et de Gaston d'Orléans, La Mesnardière affirme s'être imprégné, à force d'érudition, de toutes les connaissances historiques et rhétoriques nécessaires à une traduction fidèle dans l'esprit, et cependant très infidèle pour la lettre, quoique linéaire. Il souhaite rendre le panégyrique dans une prose inspirée, capable de ranimer l'éloquence. Mais réactualiser le discours, ce n'est pas redire à l'identique : une fonction rhétorique nouvelle ne commande-t-elle pas nécessairement cette régénération discursive ? Paru le 8 juin 1638 dans un in-quarto sans épître dédicatoire, sans nom d'auteur en page de titre, mais doté d'une longue préface, le Panégyrique de Trajan reparaît en 1642 au format in-12 avec un privilège presque identique, jusqu'à la date d'achevé d'imprimer. La seule différence concerne le statut de l'auteur, désigné sous son nom, en qualité de "conseiller et médecin de son Altesse royale» puis de «conseiller et médecin de Mgr le duc d'Orléans »: entretemps la naissance du futur roi a fait perdre à Monsieur son rang d'héritier du trône. En dehors de la traduction elle-même, seule la préface fournit au lecteur quelques éléments historiques généraux. Le texte est continu, sans sections, sans alinéas, sans marginales.

\subsection{Le choix d'une énonciation hybride}

Avant d'aborder le problème de l'instance énonciative, il faut expliquer le choix de la paraphrase. La Mesnardière multiplie par trois la longueur de ce discours publié dont il 
respecte la dispositio à de rares exceptions près : il dilate une pensée souvent concise, il relâche les mailles d'un style serré, il intègre au texte un commentaire didactique ou moral. La préface justifie avec aplomb le caractère inspiré de ce qui apparaît bien, suivant les termes d'Érasme, comme une imitatio plutôt qu'une translatio ; cette forme " plus libre de commentaire continu, sans changement de personne $»^{5}$ fait entendre une énonciation hybride, la contamination de la voix de Pline par son traducteur (Zuber 1968: 81). La Mesnardière déclare s'être identifié à Pline pour prolonger les pensées du consul par les siennes, en les adaptant, la Rome des Césars n'étant rien d'autre à ses yeux qu'une monarchie ${ }^{6}$. Cette éloquence enthousiaste cherche à ranimer l'éloge d'un prince modèle. Mais à quoi bon, si ce n'est pour lui redonner une véritable fonction rhétorique, voire politique ? Une analyse philologique du discours doit permettre de percevoir une pensée propre derrière les altérations du texte originel.

Sans décrochage énonciatif, la traduction intègre les commentaires du paraphraste. La Mesnardière endosse le je de Pline, comme le montre l'interpolation suivante :

Vous voyez bien que mon Discours prend une longue carrière ; Vous voyez bien que ma joie s'exhale ici à son aise, et que je me ravis moimême par l'agréable souvenance de tant de perfections que je remarque en ce Grand Prince?.

La représentation de la joie des Sénateurs pose exemplairement le problème de l'édition du Panégyrique sans notes historiques ni philologiques. Ce choix, qui empêche toute identification de l'édition utilisée comme des leçons retenues, confirme que La Mesnardière cherche à faire revivre un discours, non à éditer un texte :

Quels feux extraordinaires nous ont enflammé les Esprits, pour élever nos pensées à un degré digne de vous? Et pour nous fournir des paroles qui fussent de puissantes marques des biens que nous avions reçus, et du souvenir généreux qui nous en demeurait dans l'Âme? Il faut croire, Divin Monarque, que c'est votre seule Vertu qui a mis dans notre bouche ces mots expressifs et ardents que nous vous avons prononcés ${ }^{8}$.

Le paraphraste met à profit la technique oratoire de la visio, qui échauffe l'éloquence par la représentation mentale des choses absentes au point de les rendre présentes ${ }^{9}$.

\subsection{Captation par amplification}

Élève de Quintilien, Pline le jeune a déjà recours à ces différents procédés, qui pour la plupart n'entraînent pas un accroissement verbal (Macé : 2014). Dans la paraphrase, une première catégorie d'amplificatio correspond aux substituts emphatiques : une métaphore, comme « recueillir les roses parmi les pointes des épines » pour mieux rendre per adversa venisse ${ }^{10}$, l'ajout d'une épithète, " un Prince magnanime et toujours victorieux » traduisant invicti imperatoris ${ }^{11}$, le renfort des hyperboles ou des périphrases. Mais La Mesnardière procède plus volontiers encore par amplificatio rerum. La simple phrase « Non solitudinem illi [delatores], non iter, sed templum, sed forum insederant», ce n'est pas dans un lieu désert, ni sur un chemin qu'ils se sont installés, mais dans un temple, sur le Forum, amène l'amplification suivante :

Ces fléaux de la République ne se cachaient pas dans les bois, dans les affreuses montagnes, ni dans les creux des rochers. Ils avaient trouvé les moyens de s'introduire peu à peu dans les Cabinets des Princes ; qui étant toujours en alarme par les soupçons qu'ils avaient qu'on fit cabale dans la Ville pour secouer la Tyrannie, leur avaient donné la licence de se glisser effrontément jusques au pied des Autels, pour voir si on parlerait d'eux; et dans les augustes lieux, où l'on rendait la Justice, pour découvrir ce qu'on dirait touchant les mœurs du Souverain ${ }^{12}$. 
La Mesnardière conserve l'idée mais la développe : si la concision de Pline ne rend pas raison des mots templum et forum, il en interprète la cause. Les delatores investissent ces lieux pour mieux espionner les Romains (amplificatio per congeriem causarum). Un autre type de conversion semble également fécond, l'amplification par raisonnement (per ratiocinationem). Alors que Pline utilise volontiers les façons indirectes de louer, et montre la modestie de Trajan plus qu'il ne la dit, La Mesnardière ne manque jamais de rendre l'idée explicite. L'arrivée de l'empereur au Sénat, à pied, sans litière, se fait ainsi avec lenteur, ce que Pline attribue à la foule pressante. Or le traducteur extrapole deux causes relatives à la volonté de Trajan de se laisser admirer par les Romains, et d'apparaître en simple citoyen - «dans ce noble appareil, superbe par sa modestie, et haut par votre abaissement $»^{13}$. La beauté de cette «Entrée vraiment civile» résulte alors d'un puissant paradoxe, et tout au long du panégyrique, La Mesnardière insiste sur cette modestie dont il fait un puissant ressort de la majesté.

\subsection{Texte et contexte : une ambivalence politique}

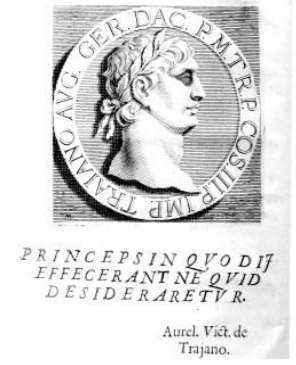

Fig. 1. Gravure de l'éd. Sommaville, 1638, $\mathrm{f}^{\circ}$ õ ij vº (CESR, Tours)

Le dépouillement de l'édition originale du panégyrique donnée par La Mesnardière n'est pourtant pas total : en guise de frontispice apparaît la gravure d'un denier daté du troisième consulat de Trajan, en buste lauré à droite (Fig. 1). Une comparaison avec les ouvrages imprimés par Antoine de Sommaville écarte l'hypothèse qu'elle proviendrait du fonds propre de l'éditeur. En revanche, on sait que la collection numismatique de Gaston d'Orléans a été enrichie par un don du marquis de Grimaud, qui avait acheté un millier de deniers d'argent figurant les empereurs, dont Trajan ; ce lot avait été prélevé sur un trésor de cinq mille pièces romaines découvert en 1627 près de Nîmes ${ }^{14}$. Sans même tenir compte du degré d'interprétation des traits de l'empereur, il est difficile d'espérer une identification formelle. Si le modèle appartenait à la collection de Monsieur, dont le legs à son neveu a entraîné la création du Cabinet des Médailles, on peut seulement supposer que c'est là qu'il se trouve encore, mais sans mention de provenance ${ }^{15}$. Attachons-nous donc plutôt à la gravure. Ne faut-il y voir qu'une simple illustration ? Ou occupe-t-elle une fonction discursive, en l'absence d'épître dédicatoire ? Une médaille gravée par Jean Varin représente Louis XIII sur l'avers et Gaston d'Orléans sur le revers, « de profil à gauche en buste nu hérö̈que de trois-quarts, drapé d'une peau de lion comme un nouvel Hercule ${ }^{16}$, peut-être à l'imitation d'une monnaie de l'empereur gaulois Postumus; la frappe remonterait au début de 1638, alors que le prince est encore l'héritier du trône. Ainsi, quel portrait faut-il supposer au revers du denier de Trajan représenté en frontispice ? La paraphrase confirmet-elle une destination politique à l'égard du protecteur nommé dans le privilège ?

La place conférée par Pline au Sénat peut faire songer à la conception de la monarchie tempérée selon Monsieur, qui fut appelé « Père de la patrie », titre donné par Pline à Trajan. Toutefois sa première attestation semble tardive ${ }^{17}$. La paraphrase permet de rapprocher le Sénat du Parlement, que le jeune prince doive « obéir aux Remontrances du Sénat », ou que l'empereur travaille conjointement avec lui «près de quarante heures [...] sans aucune 
interruption ${ }^{18}$. Enfin, les mentions du prince-mécène peuvent lui faire référence. Mais différentes interpolations de La Mesnardière autorisent une autre hypothèse ; résumons-les : appointements pour les auteurs, importance politique du théâtre, modestie du souverain, doctrine absolutiste donnent à penser que le paraphraste songe aussi à son premier protecteur, Richelieu, auquel il resta toujours fidèle. En témoigne son discours de réception à l'Académie française, en 1655, où il évoque le projet médité par le cardinal pour l'«Illustre Compagnie », mots qui désignaient déjà le Sénat à la fin de la paraphrase ${ }^{19}$. Or voici comment La Mesnardière définit l'âme royale dans le Panégyrique :

Mais une âme vraiment Royale, équitable et généreuse, qui a toujours
été maîtresse du plus grand de ses mouvements, et ne s'est jamais
permis dans une puissance absolue, que ce qui était raisonnable, c'est
proprement celle-là qui méprise les années, qui marche à l'Immortalité
par des routes infaillibles, et qui trouve des éloges dans la bouche
même de ceux qui n'ont aucun intérêt de parler à son avantage ${ }^{20}$.

Divinité, majesté, puissance, modération, ce portrait du souverain magnanime tracé en surimpression du texte de Pline révèle une conception absolutiste tempérée par la modestie. Le Panégyrique n'est plus seulement un miroir des princes; il expose un fonctionnement idéal des institutions politiques, relevant de l'absolutisme vertueux et tempéré. Pour Cardin Le Bret, «la souveraineté n'est non plus divisible, que le point en la Géométrie », mais c'est pour ajouter aussitôt qu'un «grand Roi» doit prendre avis des Parlements et des Officiers de la couronne ${ }^{21}$. De même, dans son Testament politique, Richelieu juge indispensable que le souverain reste ouvert aux conseils, grâce à sa « modestie ».

$\mathrm{Au}$ fond, La Mesnardière nous donne moins à lire Pline que sa propre lecture de Pline. En l'absence de tout balisage typographique du texte, seule une lecture juxtalinéaire continue peut faire apparaître les variantes et interpolations dues au paraphraste. Mais le rêve d'un ethos de l'orateur reconstitué grâce à l'enthousiasme en révèle au contraire le feuilletage, à l'origine des effets, involontaires ou délibérés, de brouillage énonciatif. Est-ce le client de Gaston d'Orléans, ou celui du cardinal de Richelieu, dont la voix se fait entendre par-dessus celle de Pline? Tantôt l'un, tantôt l'autre, semble-t-il : en 1636, Vincent Voiture, dans une position similaire, avait préféré ne pas publier son hommage à Richelieu $^{22}$. Mieux valait cultiver la prudence. L'important n'est peut-être donc pas de décider, mais de mettre en évidence que cette amplification au second degré ne pouvait ranimer l'éloquence impériale sans être en prise directe avec le contexte nouveau. De là cette ambivalence foncière d'un discours historique comme revivifié par son actualité politique. Cette traduction est illisible aujourd'hui sans une analyse transdisciplinaire chargée de prélever des indices philologiques, rhétoriques, énonciatifs. La Mesnardière nous avertit en outre de l'impasse que constitue l'essai d'une reconstitution énonciative du discours : réénoncer d'aussi loin, c'est énoncer à partir d'un présent qui nous rend tributaire d'une formation discursive distincte de la source.

\section{Citer : le sublime de la référence dans Athalie (1691)}

Dans le traité longtemps attribué à Longin, le sublime apparaît tel un corps indivisible ; son surgissement est un effet de la masse du discours et de ses parties ${ }^{23}$. On peut toujours détacher un fragment identifiable au summum, cependant il ne faut pas le croire autonome : préparé en amont, il relève d'un fonctionnement transphrastique qui engage moins la dispositio que la progression textuelle, même si le contexte peut suppléer à cette masse du discours (le Fiat lux n'est vraiment saisi comme sublime que par son environnement non

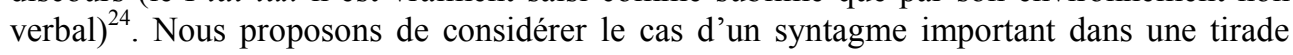
d'Athalie, car son emprunt à Bossuet, revendiqué dans la préface, est une bribe d'interdiscours nouée dans la trame d'un nouveau discours. Comment Racine l'intègre-t-il ? Ce phénomène de polyphonie énonciative engage-t-il le fonctionnement du sublime ? 
Au début d'Athalie, l'épouse du Grand prêtre Joad retrace le massacre auquel a survécu Joas, l'enfant qu'elle protège, et rappelle par ce moyen ce qui la lie au seul héritier de David, que Bossuet a nommé ce « précieux reste » :

\section{JOSABET}

Et c'est sur tous ces Rois sa justice sévère

Que je crains pour le fils de mon malheureux Frère.

Qui sait si cet Enfant par leur crime entraîné,

Avec eux en naissant ne fut pas condamné ?

Si Dieu le séparant d'une odieuse race,

En faveur de David voudra lui faire grâce ?

Hélas ! l'état horrible où le Ciel me l'offrit,

Revient à tout moment effrayer mon esprit.

De Princes égorgés la chambre était remplie.

Un poignard à la main l'implacable Athalie

Au carnage animait ses barbares Soldats,

Et poursuivait le cours de ses assassinats.

Joas laissé pour mort frappa soudain ma vue :

Je me figure encor sa nourrice éperdue,

Qui devant les Bourreaux s'était jetée en vain,

Et faible le tenait renversé sur son sein.

Je le pris tout sanglant. En baignant son visage

Mes pleurs du sentiment lui rendirent l'usage.

Et soit frayeur encore, ou pour me caresser,

De ses bras innocents je me sentis presser.

Grand Dieu, que mon amour ne lui soit point funeste.

$\mathrm{Du}$ fidèle David c'est le précieux reste.

Nourri dans ta maison en l'amour de ta Loi

Il ne connaît encor d'autre Père que toi.

Sur le point d'attaquer une Reine homicide,

À l'aspect du péril si ma foi s'intimide,

Si la chair et le sang se troublant aujourd'hui

Ont trop de part aux pleurs que je répands pour lui ;

Conserve l'héritier de tes saintes promesses,

Et ne punis que moi de toutes mes faiblesses.

\subsection{Rupture énonciative et cohérence textuelle}

Dans la deuxième scène d'Athalie, les deux tirades successives de Josabet et Joad s'achèvent par une même apostrophe à Dieu, «Grand Dieu » (v. 255 et 283), qui marque le début d'une prière tenant lieu de péroraison. Par ce décrochage énonciatif, le récit de l'histoire humaine s'interrompt sur un appel à l'aide divine, et la tirade pathétique s'élève ainsi brusquement au-dessus de la rhétorique intrascénique des personnages pour se situer à un niveau suprascénique : ce n'est plus l'un à l'autre que s'adressent Josabet et Joad, mais à Dieu. Ce mouvement ascendant amène à reconsidérer l'ensemble de la tirade, car il en détermine malgré tout la forte cohérence textuelle. L'origine et le sort de l'enfant assurent la continuité thématique. On observe en effet que la chaîne de référence assemble la tirade au moyen d'anaphores pronominales ( $i l, 1$ occurrence ; lui, 4 occ.; le / l', 4 occ.), et d'anaphores nominales, le plus souvent fidèles (" cet enfant», déterminant démonstratif à valeur pathétique ; «le fils de mon malheureux frère », «le précieux reste », « l'héritier de tes saintes promesses », périphrases emphatiques). Elles se superposent à des isotopies de la naissance et de la mort, de l'innocence et du crime dont la mise en tension accroît la violence de l'évocation ( «en naissant», « Nourri dans ta maison» / "princes égorgés », « tout sanglant»; « ses bras innocents » / « leur crime », « carnage », « assassinats »). La tirade repose ainsi sur une structuration axiologique: sa cohérence textuelle est puissamment organisée par la confrontation du Bien avec le Mal. Toutefois, le «précieux 
reste » n'est pas un élément parmi d'autres dans la chaîne de référence. Moment clé de la dispositio, dramatisé par la rime, il figure dans le premier temps de la péroraison qui fait précéder l'amplificatio d'une enumeratio, ou récapitulation : le détachement par anastrophe du complément du nom retarde la thématisation d'une périphrase à forte valeur résomptive. L'adjectif précieux concentre alors toute la force de l'hypotypose pour référer à l'héritier qui, ayant été sauvé, ne saurait l'avoir été en vain. Avec cette péroraison pathétique, la rhétorique humaine s'élève ainsi vers Dieu, dont on espère la miséricorde.

\subsection{Captation d'un segment textuel : « le précieux reste »}

Si le mot de référence a d'abord été pris au sens linguistique, il n'est pas indifférent de devoir recourir à son sens philologique pour en compléter la compréhension. Cette fois-ci, le syntagme ne relève pas d'une paraphrase intégrant le commentaire à l'énoncé originel, mais apparaît bien, quoique sans guillemets, comme une citation pouvant fonctionner aux deux niveaux de l'énonciation, soit interne, soit externe. Du reste, l'auteur a voulu faciliter un tel examen philologique, car il a choisi de livrer sa source, le Discours sur l'Histoire universelle de Bossuet: " De là vient que l'illustre et savant Prélat, de qui j'ai emprunté ces paroles, appelle Joas le précieux reste de la maison de David. Josèphe en parle dans les mêmes termes » $\left(\left[\begin{array}{lll}a & \text { iij } & v^{\circ}\end{array}\right)^{25}\right.$. Cette référence figure dans un manuscrit autographe de Racine $\left(\mathrm{BnF}\right.$, fr. $\left.12887, \mathrm{f}^{\circ} 89 \mathrm{v}^{\circ}\right)$, dont voici la transcription diplomatique :
p. 27.
$\mathrm{M}^{\mathrm{r}}$ de Meaux appelle Joas, prétieux reste de la Maison de David ${ }^{26}$
Joseph
Athalie voulut qu'il ne restast pas un seul de la maison de David, et elle crut
$\mathrm{M}^{\mathrm{r}} \mathrm{d}^{\prime}$ And. avoir exécuté son dessein, il ne resta qu'un seul, qui estoit fils d'Okosias.
$[\ldots]$ Voilà le seul qui vous reste de la Maison de David.
Le P. R. Josabet conserva Joas, po et Dieu le permit pour empescher que la race de David ne fust esteinte.

Ce ne sont pas moins de quatre références successives. Outre le passage de Bossuet, on relève une prise de notes d'après Flavius Josèphe, sa traduction par Arnauld d'Andilly, prise dans son édition des Antiquités judaïques, parue sous le titre d'Histoire des Juifs, Paris, Le Petit, 1681, IX, VII, p. 339, et enfin la paraphrase d'un verset de la Bible dans la traduction de Port-Royal («Le P. R.»), deuxième livre des Rois, IV, XI. La mention allographe en tête du folio initial de ces notes précise que Racine a dû travailler à cette tragédie entre 1689 et 1691 ; préface et tirade montrent qu'il maîtrisait de telles références. Mais Georges Forestier pense que ces notes préparaient plutôt une réplique à d'éventuelles critiques $^{27}$. Ce seraient donc des remarques philologiques de l'auteur, non seulement sur son propre texte, mais aussi sur le contexte biblique auquel il a été arrimé.

Il nous faut maintenant comprendre comment la citation de Bossuet peut être assimilée par l'énonciation dramatique tout en demeurant perceptible par le spectateur, et ce d'autant plus quand elle aura été exhibée au lecteur dans la préface. Or la périphrase « le précieux reste » apparaît à un point d'articulation du discours, entre la narration sous la forme de l'hypotypose, et la prière en guise de péroraison, ce qui coïncide avec le passage d'un sublime de l'emphase à un sublime biblique, que sa simplicité même rend peu visible :

Du fidèle David c'est le précieux reste.

Nourri dans ta maison en l'amour de ta Loi

Il ne connaît encor d'autre Père que toi.

À l'amplificatio verborum, insistance sur le mot, succède donc une amplificatio rerum qui, en donnant à voir la relation directe de l'enfant avec Dieu, participe du mouvement transcendant de la prière. Il est utile de considérer à présent la réénonciation de l'emprunt à Bossuet, qui surgit à la fin d'une tirade d'Abner, dans une citation involontaire de Josabet :

Que feriez-vous de plus, si des Rois vos aïeux

Ce jeune Enfant était un reste précieux $?^{28}$ 
En dépit d'un style sublime, l'officier n'atteint pas au sublime. Ce n'est pas la seule position de l'adjectif qui en diminue la force : l'accumulation de questions pressantes révèle une sorte de propension cornélienne à l'action qui se heurte au silence et à l'inaction du Grand Prêtre Joad. Or cette inaction qui le surprend est proprement une action sublime, verbalisée par contrecoup dans une très brève réplique, adressée à Josabet : "Il n'est pas temps, Princesse $»^{29}$. Une nouvelle tirade d'Abner confirme sa conversion et la force de sa foi, et c'est alors que Joad consent à reconnaitre que « de David un trésor est resté ${ }^{30}$.

L'hypotypose prépare l'irruption de ce sublime. La péroraison commence bien avec une enumeratio, qui ressaisit l'idée majeure de l'hypotypose : Joas, héritier de David, a survécu au massacre. Il est donc ce "précieux reste ». Mais le profit tiré par Racine de l'effet de polyphonie énonciative vise à l'édification du spectateur par le sublime. Les mots se détachent soudain de l'arrière-plan tragique et portent vers Dieu la prière de Josabet. Le sublime tient en partie à la force de cet énoncé qui traverse l'énonciation fictive pour toucher le spectateur. La préface donne cependant au lecteur à saisir l'interdiscours, moins pour rendre hommage à l'acuité de la formule de Bossuet, que pour indiquer que le sublime n'est saisi dans toute sa profondeur que par l'interaction entre un co-texte et un contexte.

On peut poser à ce stade que le repérage pertinent des catégories rhétoriques passe par une histoire de la rhétorique, ou par une autre appréhension du discours (ATD) qui en fouille le fonctionnement, mais il est possible que ces deux approches se rejoignent. Il importe moins de nommer les catégories rhétoriques à l'œuvre dans le texte, que d'objectiver leur fonctionnement discursif.

\section{Imiter : sur une transformation de la rhétorique par La Bruyère (1694)}

Dans la huitième édition des Caractères, La Bruyère ajoute au chapitre « Des biens de fortune » un fragment de harangue à la reine de Palmyre. Par sa dispositio incomplète, par la cohérence de ses marqueurs diégétiques, par le contraste entre une voix anonyme et le silence d'une allocutaire nommée dans deux vigoureuses apostrophes, cet alinéa ressemble aux bribes de discours transmis par les historiens ${ }^{31}$. La rupture avec l'environnement textuel - rupture énonciative, tonale, générique - le signale d'autant mieux à l'attention du lecteur :

II Ni les troubles, Zénobie, qui agitent votre empire, ni la guerre que vous soutenez virilement contre une nation puissante depuis la mort du Roi votre époux, ne diminuent rien de votre magnificence : vous avez préféré à toute autre contrée les rives de l'Euphrate pour y élever un superbe édifice, l'air y est sain et tempéré, la situation en est riante, un bois sacré l'ombrage du côté du couchant, les Dieux de Syrie qui habitent quelquefois la terre n'y auraient pu choisir une plus belle demeure ; la campagne autour est couverte d'hommes qui taillent et qui coupent, qui vont et qui viennent, qui roulent ou qui charrient le bois du Liban, l'airain et le porphyre; les grues et les machines gémissent dans l'air, et font espérer à ceux qui voyagent vers l'Arabie, de revoir à leur retour en leurs foyers ce Palais achevé, et dans cette splendeur où vous désirez de le porter, avant de l'habiter vous et les princes vos enfants. N'y épargnez rien, grande Reine ; employez-y l'or et tout l'art des plus excellents ouvriers, que les Phidias et les Zeuxis de votre siècle déploient toute leur science sur vos plafonds et sur vos lambris ; tracez-y de vastes et de délicieux jardins, dont l'enchantement soit tel qu'ils ne paraissent pas faits de la main des hommes ; épuisez vos trésors et votre industrie sur cet ouvrage incomparable ; et après que vous y aurez mis, Zénobie, la dernière main, quelqu'un de ces pâtres qui habitent les sables voisins de Palmyre, devenu riche par les péages de vos rivières, achètera un jour à deniers comptants cette royale maison pour l'embellir, et la rendre plus digne de lui, et de sa fortune ${ }^{32}$. 
Dans son Art d'écrire, Condillac juge la pensée mal développée, car il ne voit pas dans la clausule la suite naturelle des circonstances apportées par l'ouverture héroïque ${ }^{33}$; ce défaut de rotunditas fait manquer l'unité du bon style périodique, qui fonde le modèle esthétique, sémantique et logique du paragraphe ${ }^{34}$. La perception d'une faute amorce la textualisation du fragment qui se poursuit au siècle suivant: Gustave Lanson note que le caractère figure dans tous les Morceaux choisis de La Bruyère, mais lui n'y voit plus que «de la rhétorique pittoresque, et non morale ${ }^{35}$. Sous les froids artifices d'un texte trop emphatique, trop disparate, on peut pourtant retrouver une chaleur perdue. L'hétérogénéité constitutive du fragment en offre les moyens.

\subsection{Une harangue " asiatique et romaine ": les traces de l'interdiscours}

Sur le modèle de la déclamation, la cohérence des marqueurs diégétiques confère à l'instance énonciative la voix d'un orateur fictif. Sans exorde, les lieux du genre épidictique - vaillance et magnificence du souverain - désignent ce froid déclamateur comme un courtisan de Zénobie. La prose est à sa marque ; thématisée par l'apostrophe en incise, l'assonance en $i$ prolonge l'écho de la véhémente polysyndète qui articule la négation des accidents extérieurs - l'approche menaçante de l'ennemi ne suscite que le rejet superbe de toute faiblesse. La «magnificence » et la «fortune » sont respectivement le climax de la première période et le mot de la pointe : ils témoignent du glissement axiologique opéré de l'un à l'autre par l'hypotypose, et ils justifient les contradictions délibérées qui parcourent le fragment. C'est d'ailleurs en les suivant que l'on peut faire réapparaître le discours.

Le public lettré et le public mondain avaient toutes les raisons de bien connaître la reine de Palmyre. Au plus près de La Bruyère, ce personnage historique fait l'objet de mentions inégalement développées dans les traités de Fleury (1692), Le Nain de Tillemont (1691) ou Bossuet $(1681)^{36}$. Boileau, surtout, présente l'auteur du Traité sur le sublime comme son ministre (1674). Toutefois, de manière assez révélatrice, Zénobie doit vraiment sa notoriété à une première carrière littéraire très active au milieu du siècle : en 1647, elle est choisie par l'abbé d'Aubignac pour une tragédie en prose qui devait illustrer les règles poétiques, et elle figure dans La Galerie des femmes fortes de Le Moyne ${ }^{37}$; il la montre farouche et intrépide, et même ennemie du luxe, ce qui ne la désigne pas comme source directe de La Bruyère, sinon pour l'adverbe virilement. C'est en effet une Minerve, comme le suggère la gravure d'Abraham Bosse, donnée en pleine page (Fig. 2) :

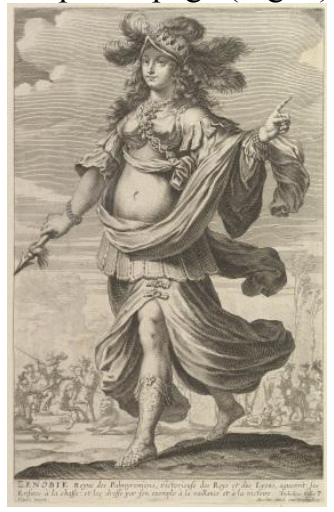

Fig. 2. Gravure de l'éd. Sommaville et Courbé, 1642, p. 144, Cl. Vignon et A. Bosse (CESR, Tours)

Mais cette notoriété mondaine trouve sa source dans Les Femmes illustres, ou les harangues hérö̈ques, de Georges et Madeleine de Scudéry (1642), recueil de vingt déclamations dédiées « Aux dames ». Prisonnière des Romains, Zénobie donne à ses filles l'exemple de la dignité d'une reine, pour montrer que la vertu n'a "point de sexe affecté » (112) : elle a suivi le char du triomphe «toute chargée de fer, ou pour mieux les nommer, de chaînes d'or et de 
pierreries, comme une illustre esclave » (122). Ce sublime de la dignité en fait un modèle d'empire sur soi-même, un an avant Cinna. Et sans doute La Bruyère se souvient-il du recueil : polysyndète remarquable dès la première phrase de Marianne à Hérode ( Ce n'est ni la crainte de la mort, ni le désir de la vie qui me font parler aujourd'hui », 19), antonomases métaphoriques des noms d'artistes qui doivent travailler à la postérité d'Alexandre ${ }^{38}$, et surtout péroraison introduite par une série de verbes à l'impératif, dans la belle harangue d'Artémise à Isocrate, où elle évoque le Mausolée qu'elle a fait édifier :

Considérez quelle est la dépense que je fais pour la structure de ce magnifique Tombeau; et jugez de là, que celle qui dépense tant de Trésors pour des Marbres muets, ne sera pas ingrate, quand vous parlerez à la Gloire de son cher Mausole ${ }^{39}$.

Dans cette première harangue, Artémise livre une réflexion sur le panégyrique : elle veut persuader Isocrate et Théopompe de consolider le Mausolée par des discours plus éternels que le marbre. Quant à La Bruyère, il emprunte ostensiblement aux Scudéry toute une phraséologie oratoire de l'éclat. L'abondance fastueuse du lexique imite le déploiement visible du palais en construction, puis le devance dans une amplification finale qui appelle à l'augmentation quasi infinie des richesses les plus difficilement concevables. Mais ce faste retourne en vanité la sagesse de l'héroïne et oratrice, comme elle la réduit au silence, renversant ainsi l'ordre des sexes établi par le dispositif scudérien.

Aucun historien ou déclamateur ne prête pourtant à Zénobie la construction d'un palais sous son règne. Chez La Bruyère, la captation convenue du discours épidictique le plus courtisan invite à parer l'éloquence d'ornements empruntés à la poésie, tant pour les images que pour le rythme et les harmoniques. Deux indices textuels font reconnaître une référence au premier livre de l'Énéide. Le héros entre dans Carthage en construction : au milieu un bois sacré y répand son ombre abondante, "Lucus in urbe fuit media, lætissimus umbra » (v. 445), puis la description s'attarde sur l'activité des ouvriers (v. 423-429). Mais ces réminiscences orientent moins la captation vers l'hommage que vers la parodie du détournement épidictique et politique des imitations de Virgile. Comme la statue de Zeuxis, cette reine de Palmyre rassemble les souvenirs d'Artémise, de Didon, de Sémiramis, et son palais remploie les marbres du Mausolée, de Carthage et de Babylone. L'interdiscours se trouve donc affiché.

Pourtant, une dernière source mondaine mérite encore d'être convoquée. Si les preuves textuelles y sont moins nettes, c'est que La Bruyère peut imiter les vers de Virgile, qui seront reconnus, tandis qu'il suffit de parodier un genre, quitte à piller la prose d'un inconnu. Dans le roman d'un certain d'Aigue d'Iffremont, Rodogune, histoire asiatique et romaine (1667), Zénobie accueille Rodogune, reine des Parthes au II siècle av. J.-C., mais n'en est pas moins dite princesse de Palmyrénie, et sa maison, située près de l'Euphrate, protégée " du côté du couchant et du nord » par l'ombre d'une forêt, donne lieu à une riche description orientale. Surtout, les campagnes voisines sont habitées par des bergers venus de Théocrite et de Virgile, et qui aiment, bien au-dessus de leur rang, des princesses :

Quand ce serait une Reine, répliqua ce beau Chasseur, cette qualité ne m'empêcherait pas de l'aimer : au contraire elle m'y animerait davantage ; et si par ma naissance il ne m'était pas permis de m'élever jusqu'à elle, je suis peut-être capable de soutenir assez dignement les sentiments qu'elle m'aurait inspirés, pour ne lui pas faire de honte quand ils viendraient à sa connaissance ${ }^{40}$.

La clausule de La Bruyère détourne ironiquement le désir d'élévation, en le soumettant à une axiologie nouvelle qui laisse affleurer la voix du moraliste. Aux troubles que méprise une femme superbement reine répond un renversement de l'ordre social, et l'on tombe ainsi du sublime héroïque dans le merveilleux romanesque. Sans doute le défaut de composition perçu par Condillac tient-il à cette contamination des modèles poétiques et romanesques. Mais il révèle que le personnage de Zénobie est reconfiguré par un discours anti-mondain. 


\subsection{De l'éloquence à l'esprit : captation et subversion des modèles}

Pour Boileau, Cassius Longin aurait engagé Zénobie à confirmer son caractère en refusant de se rendre, comme l'y invitait l'exemple de son aïeule Cléopâtre. On sait aujourd'hui que ce ministre ne pouvait être l'auteur du traité, qui n'a pas vécu sous le règne d'Aurélien, mais de Tibère. Cette erreur historique a permis d'imaginer le discours d'un orateur qui apparaît implicitement comme l'auteur du Traité sur le sublime. Plus encore que l'apostrophe et l'hypotypose, que conseille Longin, il faut remarquer l'impératif « épuisez vos trésors "), car cette abondance créant le vide apparaît proprement comme le summum : c'est par ce sursaut d'orgueil qu'il faut répondre à l'adversité. Le caractère de Zénobie semble ainsi parfaitement illustré. Cependant tout n'est pas dit. Si la magnificence est bien une qualité de souverain, elle ne saurait s'exercer selon La Bruyère au détriment de la paix, comme il est remarqué dans " Du Souverain et de la République », 24. Grâce aux circonstances, Zénobie tient l'occasion de se montrer parfaitement reine.

Mais l'orateur ne conseille nullement de bâtir un tel palais. Le trait d'esprit final est caractéristique d'une dispositio propre aux Caractères. Sans décrochage énonciatif ni lexical, il intervient de l'intérieur, moyennant un parasitage de l'instance énonciative. Soudain, il laisse poindre la voix du moraliste et provoque un changement tonal ; l'humiliation de la reine coïncide avec le passage du sublime à l'esprit. Ce que l'on pouvait savoir au XVII ${ }^{\mathrm{e}}$ siècle, une note nous l'apprend: Gourville, ancien intendant de Condé, a fait embellir le château de Saint-Maur-des-Fossés que le prince lui avait laissé. Le monde possible pointé par l'orateur serait donc imité d'une réalité contemporaine assez ironique par elle-même. Mais pour le railler sans particulariser le propos, peut-être valait-il mieux " parler de loin » (Calas : 2013), et recourir à la fiction orientale.

Quand la vanité du pâtre l'emporte virtuellement sur celle de la reine, la roue de Virgile se confond avec la roue de Fortune, et nous sommes poussés à relire l'éloge somptuaire comme un diasyrme (Tourette 2008: 332), figure dont La Bruyère paraît le «maître » (Tourette $2008^{2}: 367$ ). Maintenue par les effets de liage sémantique, la cohérence textuelle dissimulait le piège final d'une rupture énonciative et rhétorique, avec le changement apparent de visée du discours. Toute la dramaturgie morale des Caractères annonce cette « parole diogénique » (Noille-Clauzade : 2004). La pointe agit comme un inverseur d'ethos, révélant que l'orateur n'est pas le flatteur que l'on croyait, mais un ministre à la parole véhémente, dont le discours de remontrance indirect dissimule le genre délibératif sous l'épidictique : ainsi parle-t-on aux princes. L'éloge de la reine à travers celui de son palais ne joue la parfaite adaptation entre res et verba que pour mieux dénoncer le luxe.

Avec le premier temps de l'apostrophe, le processus de mythification de la dignité est saisi dans sa course, puis brutalement arrêté par les futurs prophétiques : le rachat du palais par un berger enrichi n'empêchera pas de nouveaux embellissements. Cette ironie à double détente renvoie dos à dos la magnificence des Grands et la fortune des parvenus. Dispositif redoutable, le diasyrme sert la démystification de la splendeur, car il piège le seul véritable énonciataire du caractère : en contraignant le lecteur à faire retour sur le fragment, il l'amène à confirmer la pertinence de ce blâme anti-mondain. La satire, pour être indirecte, n'en est pas moins portée par un orateur cynique et plein d'esprit. Elle vise le parvenu aussi bien que le prince, tout en disqualifiant le détournement politique du sublime. C'est que, en définitive, le seul sublime qui vaille pour La Bruyère retient la meilleure leçon de Longin et de Boileau: «Les plus grandes choses n'ont besoin que d'être dites simplement, elles se gâtent par l'emphase, il faut dire noblement les plus petites ; elles ne se soutiennent que par l'expression, le ton, la manière ${ }^{41}$ (cf. Badiou-Monferran $2000: 352-353$ ). On ne saurait confondre l'éloquence de cour avec le sublime conforme à l'idéal chrétien. Ce qui apparaissait d'abord comme une captation du discours épidictique enrôlant les vers de Virgile s'avère en fin de compte une entreprise de subversion anti-mondaine des modèles. 


\section{Conclusion}

Artificielle à nos yeux de modernes, la chaleur oratoire étudiée dans ces trois cas pourrait n'être que textuelle, ce qui la réduirait à sa dimension littéraire. Or elle signale la présence du discours dans le texte. Ces paroles vives supposent à chaque fois un ethos qui tient de la fiction. Ethos brouillé par réinvestissement de l'instance énonciative chez La Mesnardière ; ethos du personnage recommandable par sa piété, où le sublime biblique peut prendre sa source pour Racine; ethos dédoublé enfin avec La Bruyère, dans un glissement de l'instance énonciative qui diffère jusqu'à la pointe l'élucidation de la persona: ces procédures de prise en charge des énoncés construisent le cadre d'un retravail discursif, qu'il soit politique, esthétique, ou moral, de la rhétorique. De tels aménagements seraient difficiles à percevoir autrement que comme des entorses au code rhétorique, et difficiles à identifier puis analyser sans une perspective doublement ouverte à l'analyse de discours, et à la matérialité des textes. La méthode proposée cherche à compenser la perte ou l'effacement du discours, qui tient à notre différence de position; nous ne pouvons plus lire les textes comme ils étaient lus jadis, mais on cherchera à les donner à relire au plus près en ranimant le discours éteint en eux. Traduire, citer, imiter : dans ces premiers jalons, la philologie du discours est apparue comme une philologie de l'interdiscours, parce que tout discours s'énonce par rapport à d'autres discours qui le déterminent. Laissé en dépôt dans le texte, en effet, le discours a été révélé comme un précieux reste.

Tâchons pour finir de tracer les grandes lignes de ce que pourrait être une philologie du discours, en systématisant un peu notre propos. Tandis que les sciences du discours abordent surtout leur objet pour lui-même, une philologie du discours, en portant sur des textes et des discours du passé, vise à donner les moyens d'une herméneutique qui doit apparaître solidement fondée sur le double plan épistémologique (validation d'une méthode) et historique (validité des résultats obtenus par cette méthode). Cette finalité nécessite de recourir aux disciplines réunies par la philologie (paléographie, codicologie et bibliologie selon Zumthor, liste à compléter par traductologie), de concert avec les sciences du discours. Les opérations de l'auteur soumises à l'étude porteront sur une source identifiée (traduire, citer, emprunter, imiter, copier, annoter, commenter), ou mal identifiée mais perceptible (réinvestir : cadres discursifs préconstruits tels que lieux communs, stéréotypes, genres, types, ou encore lexique témoignant d'un remploi qui fait entrevoir une filiation). Mais le philologue dispose d'opérations propres, qui embrassent ou dépassent la responsabilité de l'auteur : elles concernent les supports matériels qu'il lui revient de confronter pour faire apparaître les variantes par collation de manuscrits, d'éditions successives, par convocation d'archives pouvant éclairer l'objet du travail (lettres, mémoires, registres, minutes). Enfin, reste le cas particulier du discours inaccessible directement et immédiatement, mais qui le resterait en quelque façon par des intermédiaires; il paraît possible d'envisager l'étude d'une image de soi (ethos), non plus fixée par la personne, mais par des tiers au moyen de médias variés, principalement textuels et iconiques, et laissant identifier une cohérence interdiscursive et intermédiale. Cette ouverture possible à la dimension sémiotique du discours répond encore à l'impératif de contextualisation, nécessairement interdisciplinaire. La philologie répare, restaure, rétablit. Ressaisir le discours effacé par le temps, tel serait bien l'enjeu fondamental d'une philologie du discours.

\section{Références bibliographiques}

Adam, J.-M. (2005). «Les sciences de l'établissement des textes et la question de la variation ». Sciences du texte et analyse du discours. Enjeux d'une interdisciplinarité, dir. J.-M. Adam, U. Heidmann, Genève, Slatkine Érudition, p. 69-96.

Adam, J.-M. (2011, 3 ${ }^{\mathrm{e}}$ éd.). La Linguistique textuelle. Paris : Armand Colin. 
Adam, J.-M. (2015). «L'analyse textuelle des discours, entre linguistique textuelle et analyse du discours ». L'Analyse de discours. Sa place dans les sciences du langage et de la communication dir. J.-Cl. Soulages, hommage à Patrick Charaudeau, PUR, p. 19-26.

Adam, J.-M. (2018). Le Paragraphe : entre phrases et texte. Paris : Armand Colin.

Amossy, R. (2005). «Rhétorique et analyse du discours. Pour une approche socio-discursive des textes ». Sciences du texte et analyse du discours, p. 163-177.

Amossy, R. (2015). "La place de l'influence et de la persuasion dans l'analyse du discours ». L'Analyse de discours. Sa place dans les sciences du langage et de la communication, p. 55-62.

Badiou-Monferran, C. (2000). Les Conjonctions de coordination ou "l'art de lier ses pensées » chez La Bruyère. Paris : H. Champion.

Calas, F. (2013). «Entre analyse du discours et stylistique historique. Étude de stratégies discursives pour "parler de loin" ». La Littérarité des belles-lettres. Un défi pour les sciences du texte?, dir. C. Badiou-Monferran, Classiques Garnier, p. 51-63.

Duval, F. (2007). « À quoi sert encore la philologie ? ». Laboratoire italien, 7, p. 17-40 [en ligne : https://journals.openedition.org/laboratoireitalien/128].

Espagne, M. (1997). "L'invention de la philologie. Les échos français d'un modèle allemand». Histoire Épistémologie Langage, t. 19, fasc. 1, 1997, p. 121-134.

Kerbrat-Orecchioni C. (2015). «De l'analyse du discours à l'analyse des discours ». L'Analyse de discours. Sa place dans les sciences du langage et de la communication, p. 27-36.

Macé, S. (2014). «L'amplification, ou l'âme de la rhétorique. Présentation générale ». Exercices de Rhétorique, $\mathrm{n}^{\circ} 4$ [en ligne : http://rhetorique.revues.org/364].

Maingueneau, Dominique (2005). «Philologie et analyse du discours». Sciences du texte et analyse du discours. Enjeux d'une interdisciplinarité, p. 37-49.

Maingueneau, D., Charaudeau, P. (2002). Dictionnaire d'analyse du discours, Paris : Seuil.

Maingueneau, D. (2012), «Que cherchent les analystes du discours?», Argumentation et Analyse du Discours, 9. [en ligne : https://journals.openedition.org/aad/1354]

Noille-Clauzade, C. (2004). L'Éloquence du sage. Platonisme et rhétorique dans la seconde moitié du XVII siècle. Paris : Champion.

Rastier, F. (2001). Arts et sciences du texte. Paris : PUF.

Rastier, F. (2001). "Sémiotique et sciences de la culture ». Linx, 44, p. 149-168 [en ligne: https://journals.openedition.org/linx/1058]

Tourrette, É. (2008). Les Formes brèves de la description morale. Paris : Champion.

Tourrette, É. $\left(2008^{2}\right)$. «La Bruyère et la science des détails ». Discours politique et genres littéraires: $X V I^{e}-X V I I^{e}$ siècles, dir. S. Gruffat, O. Leplâtre, p. 353-372.

Zuber, R. $(1968,1995)$. Les « belles infidèles » et la formation du goût classique. Paris : Albin Michel.

Zumthor, P. «Philologie ». Encyclopcedia Universalis. [http://www.universalis-edu.com/encyclopedie/philologie/]

\footnotetext{
${ }^{1}$ Longin, Traité sur le Sublime, éd. F. Goyet, trad. Boileau, Paris, LGF, 1995, XVI, p. 105.

${ }^{2}$ Boileau, Art poétique, III, v. 301.

${ }^{3}$ Les textes sont tous repris d'après les éditions originales. La ponctuation et les majuscules sont conservées, et pour des raisons de lisibilité, l'orthographe est modernisée, sauf mention contraire.

${ }^{4}$ Pline le jeune, Lettres, éd. H. Zehnacker, N. Méthy, Paris, Les Belles Lettres, t. III, VIII-IX, 2012.

${ }^{5}$ Érasme, Opus epistolarum, éd. Allen, Oxon, Clarendon, 1924, t. V, Ep. 1274, 1. 37-39, p. 47.

${ }^{6}$ Panégyrique de Trajan, Paris, Antoine de Sommaville, 1638, «Préface », fo õ ij (ensuite Pan. Tr.).

${ }^{7}$ Pan. Tr., p. 89 (cf. Pline, XXV § 1).
} 
${ }^{8}$ Pan. Tr., p. 281-282 (cf. Pline, LXXII § 5).

${ }^{9}$ Quintilien, Inst. orat., VI, II, § 29-30.

${ }^{10}$ Pan. Tr., p. 155 (cf. Pline, XLV § 1).

${ }^{11}$ Pan. Tr., p. 27 (cf. Pline, VIII § 2).

${ }^{12}$ Pan. Tr., p. 118 (cf. Pline, XXXIV).

${ }^{13}$ Pan. Tr., p. 80-81 (cf. Pline, XXIII § 2).

${ }^{14}$ M.-L. Berdaux-Le Brazidec, "À propos de la collection numismatique de Gaston d'Orléans », Bulletin de la société française de numismatique, $64^{\mathrm{e}}$ année, $\mathrm{n}^{\circ}$ 9, nov. 2009, p. 229-232.

${ }^{15}$ Numérisées dans Gallica, les monnaies du département des Monnaies, médailles et antiques de la $\mathrm{BnF}$ sont trop nombreuses pour garantir une identification (voir par ex. IMP-2782).

${ }^{16}$ Gaston d'Orléans Prince rebelle et mécène, dir. J.-M. Constant, P. Gatulle, PUR, 2017, p. 238.

${ }^{17}$ Théophraste Renaudot en serait la source dans la Gazette du 9 décembre 1650 (J.-M. Constant, Gaston d'Orléans, Paris, Perrin, 2013, p. 319). Mais Richelieu s'est vu décerner le même titre par La Milletière dans un Discours des moyens d'établir une paix en la chrétienté par la réunion de l'Église prétendue réformée à l'Église romaine, Paris, s. n., 1635, p. 27, et un panégyrique anonyme l'a comparé à Trajan pour sa vertu supérieure aux autres hommes (Panégyrique à Monseigneur le cardinal de Richelieu sur le sujet du philosophe indifférent, Paris, Courbé, 1641, p. 45-46).

${ }^{18}$ Pan. Tr., p. 35 (10 § 2, au lieu de l'« approbation du Sénat»), et p. 293-294 (76 § 1).

${ }^{19}$ Pan. Tr., p. 283 et $293(73 \S 1,75 \S 5)$.

${ }^{20}$ Pan. Tr., p. 208-209. Voir également p. 319-320 (83 § 6).

${ }^{21}$ Le Bret, De la Souveraineté du Roi, Paris, Toussaint du Bray, 1632, I, ch. IX, p. 71.

${ }^{22} \mathrm{P}$. Gatulle, Gaston d'Orléans : entre impatience du pouvoir et mécénat, Paris, Champ Vallon, 2012, p. 185.

${ }^{23}$ Longin, Traité sur le Sublime, éd. citée, p. 130-131.

${ }^{24}$ Longin, éd. citée, VII, 9.9, p. 86.

${ }^{25}$ Note marg. : «M. de Meaux ». La citation complète est la suivante : « Le seul Joas fils d'Ochosias, enfant encore au berceau, fut dérobé à la fureur de son aïeule. Jézabeth sœur d'Ochosias, et femme de Joïada souverain Pontife, le cacha dans la maison de Dieu, et sauva ce précieux reste de la maison de David » (1681, Paris, Mabre-Cramoisy, Première partie, Sixième époque, p. 27).

${ }^{26}$ L'orthographe de l'adjectif « prétieux » est conservée dans l'édition originale d'Athalie.

${ }^{27}$ G. Forestier, dans Racine, Euvres complètes, t. II, Paris, Gallimard, 1999, p. 1750.

${ }^{28}$ Racine, Athalie, V, 2, v. 1625-1626.

${ }^{29}$ Ibid., v. 1628.

${ }^{30}$ Ibid., V, 2, v. 1649.

${ }^{31}$ G. Molinié décrivait ainsi les choix énonciatifs : «L'allocution avec apostrophe et marqueurs du discours direct verbo-personnels obligent à situer l'ensemble en niveau II, avec un actant émetteur masqué. L'effet est donc doublement saisissant pour le récepteur lecteur (R I), d'autant plus que l'entourage énonciatif est absolument vide et le voisinage discursif entièrement étranger » (« Les modalités énonciatives dans Les Caractères de La Bruyère », L'Information Grammaticale, ${ }^{\circ}{ }^{47}$, 1990, p. 17-18).

${ }^{32}$ Texte établi d'après l'édition originale, Paris, É. Michallet, 1694, p. 261-263. Marc Escola précise que la main en marge désigne une addition dans l'éd. VIII (La Bruyère. II. Rhétorique du discontinu, Paris, H. Champion, 2001, p. 27).

${ }^{33}$ Condillac, Cours d'étude pour l'instruction du prince de Parme [...], Parme, Imprimerie royale, 1775, De l'Art d'écrire, II, ch. I, « Des accessoires propres à développer une pensée », p. 140-142.

${ }^{34}$ Sur Condillac, cf. Marc Arabyan Le Paragraphe narratif (1994), in Adam (2018 : 20).

${ }^{35}$ G. Lanson, L'Art de la prose (1908), Paris, La Table Ronde, « La petite Vermillon », 1996, p. 148.

${ }^{36}$ Fleury, Histoire ecclésiastique, Paris, Aubouyn, Émery, Clousier, t. II, 1. VIII, 1692, p. 407-408 et 423 ; Le Nain de Tillemont, Histoire des Empereurs, Paris, Charles Robustel, t. III, 1691, p. 515-517 et 745-746 ; Bossuet, Discours sur l'Histoire universelle, Paris, Mabre-Cramoisy, 1681, p. 107-108.

${ }^{37}$ Le Moyne, La Galerie des femmes fortes Paris, Antoine de Sommaville, 1647, p. 145-153; $4^{\mathrm{e}}$ éd., 1663 , p. 201-212, $6^{\mathrm{e}}$ éd. en 1668.

${ }^{38}$ Georges et Madeleine de Scudéry, Les Femmes illustres, ou les harangues hérö̈ques, avec les véritables portraits de ces hérö̈nes, tirés des médailles antiques, Paris, Sommaville, Courbé, 1642, p. 81.

${ }^{39}$ Ibid., p. 14.

${ }^{40}$ D'Aigue d'Iffremont, Rodogune, histoire asiatique et romaine, Paris, Loyson, 1667, p. 180-181.

${ }^{41}$ La Bruyère, Les Caractères, éd. E. Bury, Paris, LGF, 1995, V, 77, p. 256. 